\title{
The pre-albumin fraction: A useful parameter in the interpretation of routine protein electrophoresis
}

\author{
R. I. HARRIS AND J. KOHN \\ From the Department of Chemical Pathology, Westminster Hospital Teaching Group, \\ Queen Mary's Hospital, London
}

SYNOPSIS A technique of protein electrophoresis suitable for routine use which ensures good separation of the prealbumin fraction is described. The clinical value of visually estimating this protein fraction from the electrophoretic strip is shown with reference to 134 cases. Low levels of prealbumin were found to be associated with liver disorders, malignancy, collagen diseases, congestive cardiac failure, and burns. Finally, pitfalls in electrophoretic technique which may cause failure to visualize the prealbumin fraction are discussed.

Prealbumin is a protein of molecular weight 61000 (Schultze and Heremans, 1966a) with a half-life in the serum of 1.9 days (Oppenheimer, Surks, Bernstein, and Smith, 1965). It has similar functions to the main albumin fraction but is of especial importance in the binding of tetra-iodothyronine and triiodothyronine (Ingbar, 1958).

In 6000 paper electropherograms of both normal and pathological sera, Riva failed to demonstrate the prealbumin fraction in a single case (Riva, 1957). However, if cellulose acetate membrane (CAM) is used as the support medium prealbumin may be seen as a definite band anodic to the main albumin fraction and clearly distinguishable from it. With careful adjustment of the sample volume, $\mathrm{pH}$, etc, two prealbumin fractions may be seen. One of these is the so-called tryptophan-rich prealbumin (Schultze Schönenberger, and Schwick, 1956), and the other is said to be an $\alpha_{1}$-acid glycoprotein (Schultze and Heremans, 1966b), but the literature on this point awaits clarification. For the purpose of this study, the prealbumin is considered as a single fraction.

Since cellulose acetate is now in common use as a support medium for routine electrophoresis in many hospital laboratories, it seems worthwhile to assess the value of visually estimating the prealbumin fraction. This 'Cinderella' protein has been neglected in routine work despite several reports and papers suggesting its diagnostic and prognostic value. In particular the work of Aly and his colleagues (Aly and Niederhellmann, 1958; Aly and Bohner, 1966; Aly, 1965) drew attention to the diagnostic value of Received for publication 5 September 1974. low prealbumin levels and our own results agree substantially with their findings.

\section{Materials and Methods}

TECHNIQUE OF ELECTROPHORESIS

In this laboratory electrophoresis is carried out witis constant currert on cellulose acetate membrane using an 0.05-0.07 $\mathrm{M}$ barbitone buffer with a $\mathrm{pH}$ of 8.6 (Kohn, 1958). To obtain a separation pattern of adequate length for the visualization of the prealbumin a bridge gap of $6 \mathrm{~cm}$ is needed. The samples are applied onto the centre of the sheet equidistant from the anode and cathode bridges. Care must be taken not to overload the membrane while applying the sample as this will cause low protein levels to appear falsely normal or even high. The use of a multiapplicator (Kohn, 1967) permits the simultaneous electrophoresis of 10 or 16 sera under standard and reproducible conditions. Bromopheno blue is used as a marker of the albumin fraction and the electrophoresis terminated when the stained band is approximately $1-1.5 \mathrm{~cm}$ away from the bridge on the anode side. Under these conditions the prealbumin will not migrate into the wick and will be visible on staining. This is done using Ponceau-S. Excellent visualization of the prealbumin may be obtained after staining with Nigrosine, but the high sensitivity of this stain makes it unsuitable for the purpose of visual quantitation. After staining the strips are dried and visually inspected. The use of a multiapplicator allows a pooled normal control serum to be inserted after every second test sample. If this is done, every test sample is situated next to a 


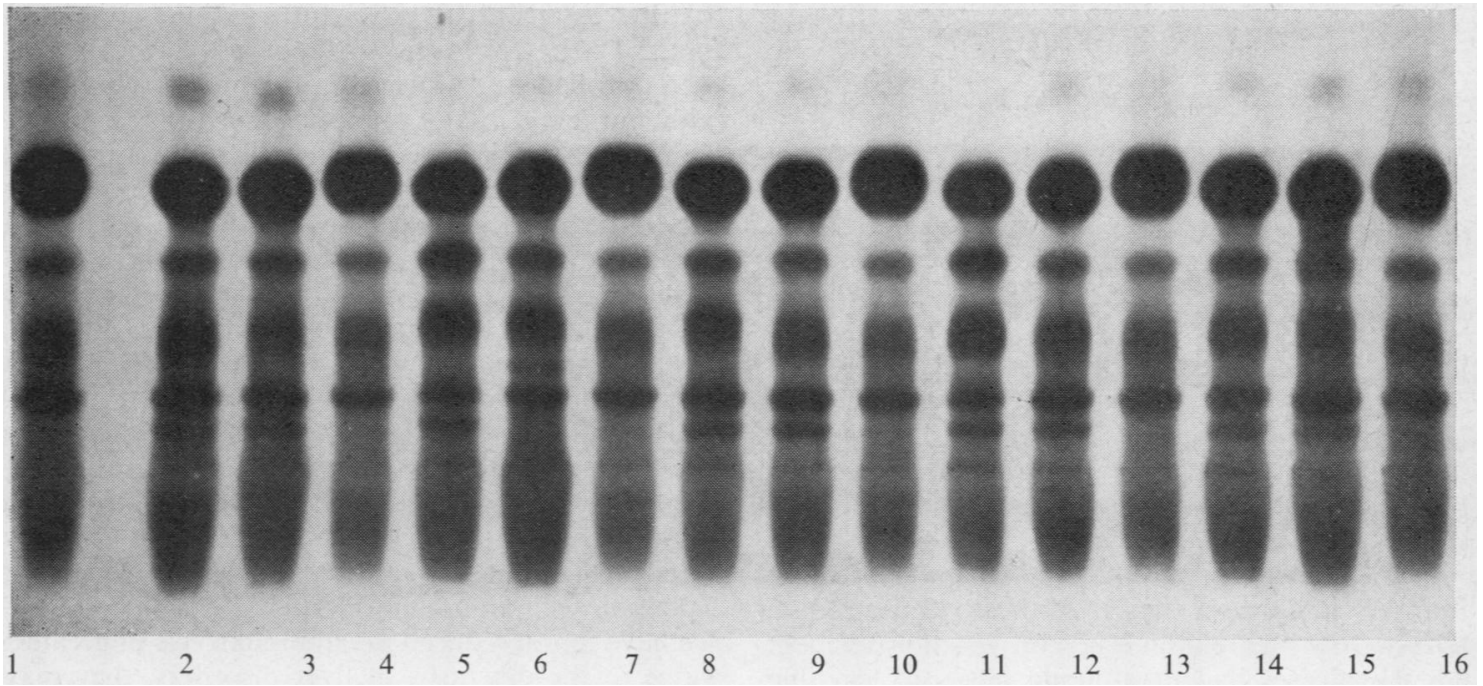

Fig 1 Photograph of typical separation pattern obtained using the techniques described. The prealbumin fraction can be clearly seen. It is absent in case 11 and equivocal in case 5.

control. Inspection against a strong light source permits ready identification of the prealbumin fraction, and the assessment of any abnormal increase or decrease in intensity. A typical electrophoretic run with the prealbumin band clearly visible is shown in figure 1.

\section{ALBUMIN DETERMINATION}

Serum albumin was determined on all samples using an automated bromcresol green dye binding method.

THE INCIDENCE OF REDUCED PREALBUMIN LEVELS IN A HOSPITAL POPULATION

A retrospective survey was made of the electrophoretic separations performed by the routine Chemical Pathology Department over an 18-month period. Those showing an unequivocal reduction or absence of the prealbumin fraction on visual inspection were followed up and the final diagnosis was obtained wherever possible.

\section{THE INCIDENCE OF REDUCED PREALBUMIN} LEVELS IN A NORMAL POPULATION

Sera from 4846 apparently healthy and symptomfree blood donors were subjected to electrophoresis and the prealbumin fraction was assessed as previously described.

CORRELATION OF REDUCED PREALBUMIN LEVELS WITH QUANTITATIVE DATA

In order to determine the sensitivity (detection level) of the inspection procedure, the degree of correlation between prealbumin assessed on an electrophoretic strip and prealbumin levels as determined by the radial immunodiffusion technique was measured. A set of sera from patients with disorders known to be associated with low prealbumin levels was selected and electrophoresed by a technician not taking part in the survey. A person who had no knowledge of the exact diagnosis in any particular case visually assessed the prealbumin levels. Immunological quantitation was then performed.

\section{Results}

The total number of patients with low prealbumin levels on whom we were able to obtain reliable clinical information was 134 . They were arbitrarily divided into five broad diagnostic categories, as follows: (1) liver disease, including cirrhosis of all types, both active and quiescent, chronic active hepatitis, and acute viral hepatitis; (2) inflammatory disorders, including the collagen disorders, acute and chronic bacterial infections and other diseases of an inflammatory nature, such as ulcerative colitis and Crohn's disease; (3) malignant disease, including the myeloproliferative disorders and the reticuloses; (4) burns; and (5) congestive cardiac failure.

The number of cases falling into each of the categories, together with the number of cases with low prealbumin and normal albumin levels are shown in the accompanying table.

Of 4846 blood donors, assumed to be clinically 


\begin{tabular}{lll}
\hline Diagnostic Category & \multicolumn{2}{l}{ Number of Cases } \\
\cline { 2 - 3 } & Total & Albumin $>3 \cdot 4$ g/dl \\
\hline Liver disease & 51 & 15 \\
Inflammatory conditions & 38 & 11 \\
Malignant disease & 31 & 7 \\
Burns & 5 & 0 \\
Congestive cardiac failure & 9 & 3 \\
\hline
\end{tabular}

Table Distribution of cases having low prealbumin levels between the diagnostic categories ${ }^{1}$

'The number of cases in each category which had normal albumin levels is also shown.

normal, only $15(0.3 \%)$ were found to have a reduced prealbumin level as judged by visual inspection of the electrophoretic strip.

The prealbumin level was determined quantitatively in 48 cases (fig 2). Of these, 13 were found to have low prealbumin levels on visual assessment. All these cases had piealbumin levels of less than $15 \mathrm{mg} / 100 \mathrm{ml}$ and all but two had levels of less than $10 \mathrm{mg} / 100 \mathrm{ml}$, (the lower limit of normal for the method in this laboratory). Thirty-three cases were found to have normal or equivocal prealbumin levels, as evaluated by inspection of the electrophoretic strip and of these only three cases were below $10 \mathrm{mg} / 100 \mathrm{ml}$. These results are statistically significant $(P<0.025)$.

The assumption that a valid assessment of a low level of prealbumin can be made by the visual inspection of an electrophoretic strip was found to be justified if fairly strict objective criteria are applied. Observer bias was eliminated as far as possible in the quantitative correlation experiment

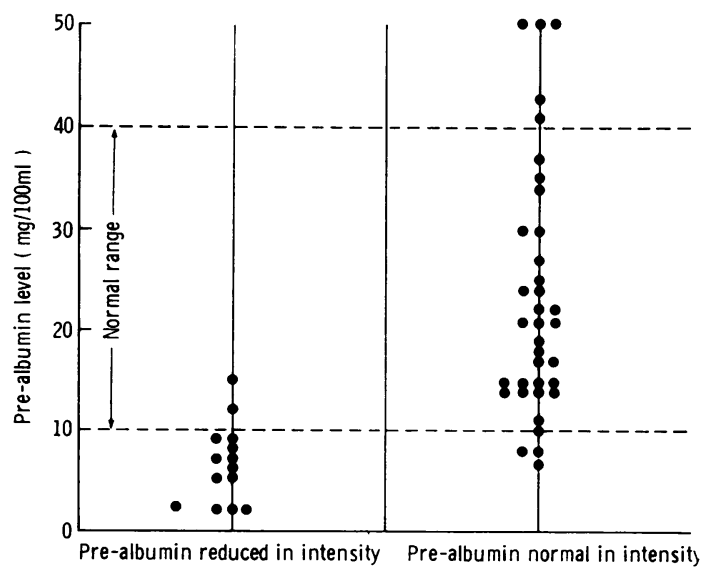

Fig 2 Correlation of quantitated prealbumin levels with visual inspection of the electrophoretic strip. It can be seen that in the majority of cases an observer can distinguish low from normal levels. by the insertion of a relatively large number of $D$ normal strips for inspection and by the fact that the $\frac{\mathscr{N}}{5}$ observer had no knowledge of the quantitative prealbumin levels in any of the specimens.

Diseases of the liver accounted for more $(38 \%) \stackrel{\text { S }}{+}$ cases of reduced prealbumin levels than any of the other diagnostic categories. This is not surprising $\overline{\bar{\omega}}$. since the liver is the site of prealbumin synthesis and $\frac{\sigma}{\sigma}$ hepatic damage of almost any aetiology may be $\stackrel{\varnothing}{\circ}$ expected to cause reduced synthetic activity. In $30 \%$ of the cases of liver damage the prealbumin was low $\overrightarrow{0}$ in spite of a normal (> $3.4 \mathrm{~g} / \mathrm{dl})$ albumin concentration. This suggests that the prealbumin may be a $\vec{\omega}$ more sensitive and perhaps earlier indication of damage to the organ than is albumin concentration alone. Other authors have made similar observations $N$ (Hallen and Laurell, 1972; Agostoni, Marasini, $\vec{N}$ Stabilini, Del Ninno, and Pontello, 1974). Although we have found reduced prealbumin levels of greatest $\mathscr{\infty}$ value in the diagnosis of liver disorders, they may 을 also be a useful pointer to inflammatory or malig- nant conditions. The reduced levels found in congestive cardiac failure almost certainly reflect $\overbrace{\infty}^{\infty}$ hepatic congestion. A point worth noting is that a $\frac{\mathbb{O}}{3}$ reduced prealbumin level in the presence of a normal or raised total protein concentration is suggestive of a hepatic aetiology; on the other hand, if the totgl $\overrightarrow{0}$ protein is low, then some form of tissue breakdow ie, chronic infection, malignancy, cachexia, etc, likely to be taking place. The extremely smati percentage of low prealbumin levels seen in clinically normal blood donors indicates that a reduced level is only very exceptionally seen in normal people. It $\stackrel{\odot}{\propto}$ may well be that some of these donors with low pre- $\overrightarrow{\vec{F}}$ albumin had pathological conditions unknown to $\frac{0}{3}$ them at the time. Our results certainly emphasize the fact that as an incidental finding reduced levels should be taken seriously and further investigations undertaken.

The technique required for the visualization of prealbumin is simple and does not require any special apparatus or skill. In fact, it is exactly the $ᄋ$ same as that necessary for a good clear electro- $₹$ phoretic separation of other serum protein fractions. 윽 In some laboratories, electrophoretic strips are $>$ scanned in a densitometer without being visually inspected. Levels of prealbumin are too low to allow accurate densitometric quantitation, and on a scan it often merges imperceptibly into the baseline. $N$ More useful information is gained by visual com- స్ట parison of the strips with a normal control serum than by densitometric scanning. The actual electro- 0 phoretic technique itself is most important. Allowing insufficient time for separation or the use of buffer $\stackrel{\mathcal{P}}{?}$ solutions of too high molarity will cause a short 7 run and failure of the prealbumin to separate from 
the main albumin fraction. If the current used is too high, for instance in inadequately cooled constant voltage systems, the same problem may arise. Failure to change the buffer in the tank frequently will cause evaporation and a consequent increase in molarity. An excessively prolonged time of electrophoresis or too short a bridge gap may cause the prealbumin to migrate into the wick and thus be lost. The use of bromophenol blue, as already described, will prevent this. Ideally, the separation patterns should be at least 3-4 cm long. Unfortunately, some electrophoretic systems in common use at present give separation patterns shorter than this and are therefore quite unacceptable for the purpose.

We are indebted to Miss $\mathbf{H}$. Joyce and Miss $\mathbf{J}$. Sabbat for technical assistance.

\section{References}

Agostoni, A., Marasini, B., Stabilini, R., Del Ninno, E., and Pontello, M. (1974). Multivariate analysis of serum protein assays in chronic hepatitis and postnecrotic cirrhosis. Clin. Chem., 20, 428-429.
Aly, F. W. (1965). Das Tryptophanreiche Prealbumin. Tübingen.

Aly, F. W., and Bohner, J. (1966). Prot. biol. Fluids, 14, 693.

Aly, F. W., and Niederhellmann, K. H. (1958). Untersuchungen zum Präalbumin im menschichen serum. I. Methodische Angaben zum papierelektrophoretischen Nachweis des Präalbumins auf Filterpapier: quantitative Veränderungen des Präalbumins. Klin. Wschr., 36, 954-959.

Hällén, J., and Laurell, C. B. (1972). Plasma protein pattern in cirrhosis of the liver. Scand. J. clin. Lab. Invest., 29, Suppl. 124, $97-101$.

Ingbar, S. H. (1958). Pre-albumin: a thyroxine-binding protein of human plasma. Endocrinology, 63, 256-259.

Kohn, J. (1968). Cellulose acetate electrophoresis and immunodiffusion techniques. In Chromatographic and Electrophoretic Techniques, edited by I. Smith, 2nd ed., vol. 2, pp. 84-146. Heinemann, London.

Kohn, J. (1967). A multi-sample applicator for zone electrophoresis. Clin. Chim. Acta, 18, 65-68.

Oppenheimer, J. H., Surks, M. I., Bernstein, G., and Smith, J. C. (1965). Metabolism of iodine-131-labelled thyroxine-binding prealbumin in man. Science, 149, 748-751.

Riva, G. (1957). Das Serumeiweissbild, p. 309. Huber, Berne.

Schultze, H. E., and Heremans, F. F. (1966a). Molecular Biology of Human Proteins, vol. 1, p. 430. Elsevier, Amsterdam.

Schultze, H. E., and Heremans, F. F. (1966b). Molecular Biology of Human Proteins, vol. 1, p. 176. Elsevier, Amsterdam.

Schultze, H. E., Schönenberger, M., and Schwick, G. (1956). Über ein Präalbumin des menschlichen Serums. Biochem. Z., 328, 267-284. 